\title{
Lake Tahoe Interagency Monitoring Program: Tributary Sampling Design, Sites, and Periods of Record
}

\section{INTRODUCTION}

Lake Tahoe is a natural resource famous for its alpine setting and deep, clear waters. Reported decreases in water clarity of about 1 foot per year (Goldman and Byron, 1986) have prompted major efforts to preserve the water quality of Lake Tahoe. This decrease in clarity is attributed mainly to human activities, which have increased dramatically in the Lake Tahoe Basin since 1960.

Increased nutrient concentrations within Lake Tahoe are considered the primary cause of increased algal growth and resultant loss of clarity in the lake (Goldman and Byron, 1986). Suspended sediment delivered to the lake by tributaries also is of concern, because some nutrients, such as organic nitrogen and phosphorus, tend to be transported with sediment particles. Tributary streamflow in the Lake Tahoe Basin is suspected of being one of the major pathways for nutrient and sediment transport to the lake (Goldman and Byron, 1986). Increased development has accelerated this transport through urbanization of wetland areas and erosion from construction on moderate to steep slopes within the basin (Byron and Goldman, 1989).

Public concern over water-quality degradation in Lake Tahoe has led to extensive monitoring activities in the lake and its tributary watersheds. One of the largest monitoring activities is the Lake Tahoe Interagency Monitoring Program (LTIMP). The purpose of this report is to describe the data collection activities of LTIMP.

\section{LAKE TAHOE INTERAGENCY MONITORING PROGRAM, 1978-87}

Since the early 1970's, prior to the formation of LTIMP, the Tahoe Regional Planning Agency (TRPA), the U.S. Geological Survey (USGS), the Tahoe Research Group (TRG) of the University of California, Davis, and State and local agencies had been monitoring the Lake Tahoe Basin for lake and tributary water-quality conditions. In 1978, LTIMP was formed to conduct collaborative monitoring and research efforts in the Lake Tahoe Basin. Participants of LTIMP include TRPA, USGS, TRG, U.S. Forest Service, U.S. Environmental Protection Agency, California State Water Resources Control Board, Lahontan Regional Water Quality Control Board (RWQCB), California Department of Water Resources, California Department of Transportation, California Air Resources Board, California Department of Fish and Game, Nevada Division of Environmental Protection, and local counties and cities. LTIMP monitoring began in the 1979 water year $^{1}$ as a cooperative program with TRG and USGS and included four tributary sites in four Lake Tahoe Basin watersheds. The program was expanded to seven sites in seven watersheds in 1981 and continued through 1987 with various sites sampled. Tributary site locations are shown in figure 1. An overview of tributary sites for LTIMP is shown in figure 2.

\section{LAKE TAHOE INTERAGENCY MONITORING PROGRAM, 1988-98}

The current LTIMP tributary-monitoring program, a cooperative effort by TRPA, USGS, and TRG, began in the 1988 water year. The primary purpose of this program is to provide long-term data on tributary water quality. This program initially included 4 sites in 4 water-

${ }^{1}$ Water year is the 12-month period from October 1 through September 30, and is designated by the year in which the period ends (for example, water year 1979 began October 1, 1978, and ended September 30, 1979).

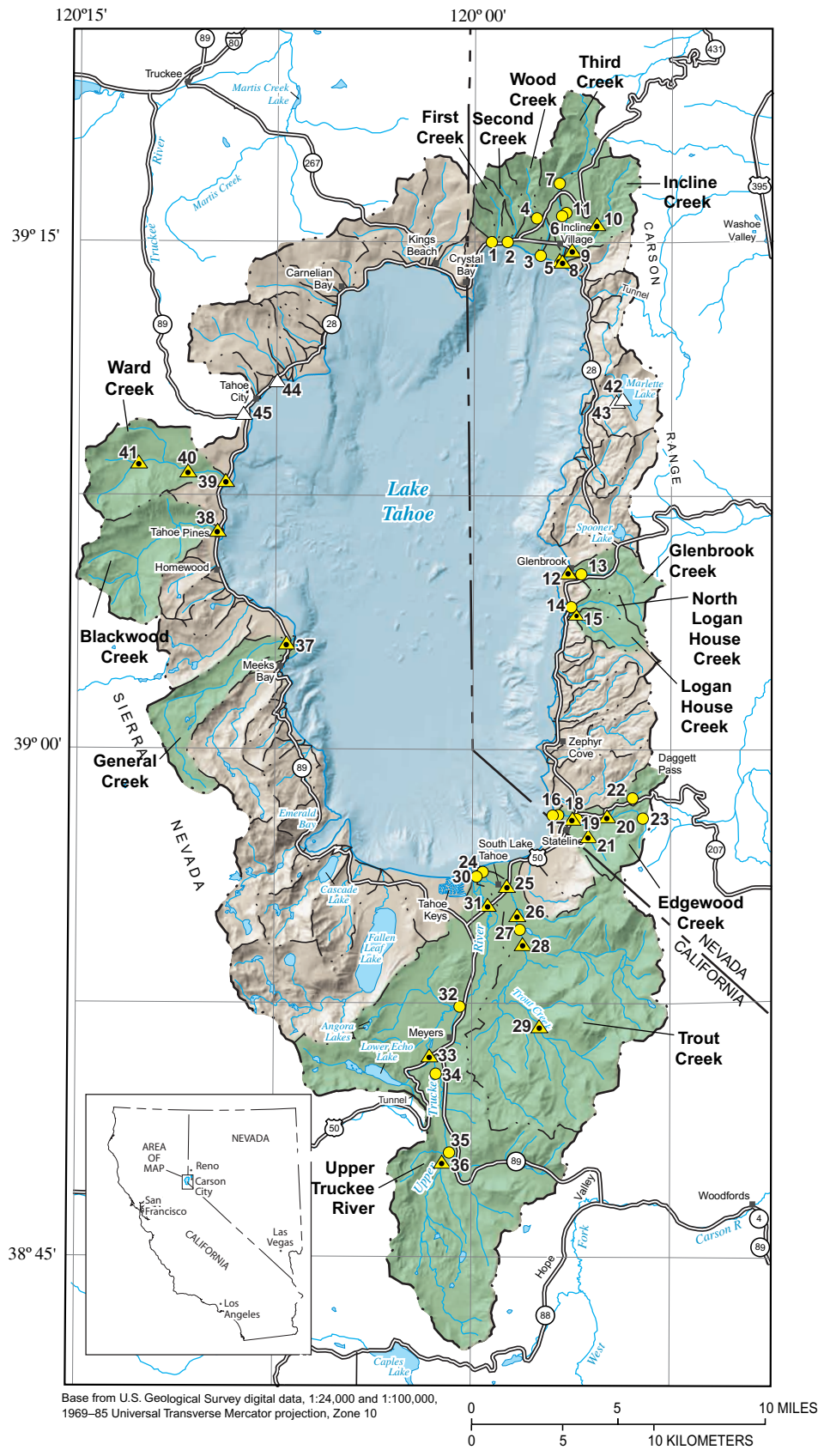

EXPLANATION

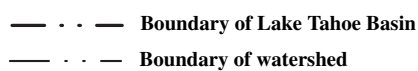

Lake Tahoe Interagency Monitoring Program monitoring station and number 10 Surface-water site and gage $42 \triangle$ Other active gage

$35 \bigcirc$ Other water-quality site

Figure 1. Geographic setting, hydrologic basins, and Lake Tahoe Interagency Monitoring Program sites in Lake Tahoe Basin, California and Nevada. 
Table 1. Periods of record for streamflow, physical, nutrient, and sediment data collected at current U.S. Geological Survey/Lake Tahoe Interagency Monitoring Program stream network sites and other active USGS gaging stations in the Lake Tahoe Basin, California and Nevada

[Abbreviations: nr, near; abv, above; ck, creek, St., street; Dr., drive; Ave., avenue; Hwy., highway; —, not collected]

\begin{tabular}{|c|c|c|c|c|c|c|c|}
\hline $\begin{array}{l}\text { Site } \\
\text { no. }\end{array}$ & Site name & $\begin{array}{l}\text { Station } \\
\text { no. }\end{array}$ & $\begin{array}{l}\text { Site } \\
\text { type }^{1}\end{array}$ & Streamflow ${ }^{2}$ & Physical $^{3}$ & Nutrients ${ }^{4}$ & Sediment ${ }^{5}$ \\
\hline \multicolumn{8}{|c|}{ Lake Tahoe Interagency Monitoring Program Sites } \\
\hline 1 & $\begin{array}{l}\text { First Creek (at Hwy. 28) nr Crystal } \\
\text { Bay, Nev. }\end{array}$ & 10336688 & $\mathrm{~m}$ & misc: $70-73,91$-current & field: 70-73,91-current & $\begin{array}{l}\text { n,p: } 70-73,79-81 \\
\text { n,p,i: } 91-\text { current }\end{array}$ & $\begin{array}{l}\text { tot: } 70-73,80,81 \\
\text { sus: } 91 \text {-current }\end{array}$ \\
\hline 2 & $\begin{array}{l}\text { Second Creek at Lakeshore Dr. nr } \\
\text { Incline Village, Nev. }\end{array}$ & 10336691 & $\mathrm{~m}$ & misc: 91-current & field: 91-current & n,p,i: 91 -current & sus: 91-current \\
\hline 3 & $\begin{array}{l}\text { Wood Creek at Lakeshore Dr. nr } \\
\text { Incline Village, Nev. }\end{array}$ & 10336694 & $\mathrm{~m}$ & misc: $70-73,91$-current & field: 70-73,91-current & $\begin{array}{l}\text { n,p: } 70-73 \\
\text { n,p,i: } 91 \text {-current }\end{array}$ & $\begin{array}{l}\text { tot: } 70-73 \\
\text { sus: } 91 \text {-current }\end{array}$ \\
\hline 4 & $\begin{array}{l}\text { Wood Creek above Jennifer St. nr } \\
\text { Incline Village, Nev. }\end{array}$ & 10336692 & $\mathrm{~m}$ & misc: 91 -current & field: 91-current & n,p,i: 91 -current & sus: 91-current \\
\hline 5 & $\begin{array}{l}\text { Third Creek (at Lakeshore Dr.) nr } \\
\text { Crystal Bay, Nev. }\end{array}$ & 10336698 & $\mathrm{~b}$ & $\begin{array}{l}\text { gage: } 70-73,75 \text {, } \\
77 \text {-current }\end{array}$ & field: $70-73,78-85,88$-current & $\begin{array}{l}\text { n,p: } 70-73,80-87 \\
\text { n,p,i: } 88 \text {-current }\end{array}$ & $\begin{array}{l}\text { tot: } 70-73,81-85 \\
\text { sus: } 88 \text {-current }\end{array}$ \\
\hline 6 & $\begin{array}{l}\text { Third Creek at Village Blvd. at Incline } \\
\text { Village, Nev. }\end{array}$ & 103366965 & $\mathrm{~m}$ & misc: 89,91 -current & field: 89,91-current & $\begin{array}{l}\text { n.p,i: } 89 \\
\text { 91-current }\end{array}$ & $\begin{array}{l}\text { sus: } 89 \text {, } \\
91 \text {-current }\end{array}$ \\
\hline 7 & $\begin{array}{l}\text { Third Creek below unnamed tributary } \\
\text { nr Incline Village, Nev. }\end{array}$ & 103366958 & $\mathrm{~m}$ & misc: 89,91 -current & field: 89,91-current & $\begin{array}{l}\text { n,p,i: } 89 \text {, } \\
\text { 91-current }\end{array}$ & sus: 89,91 -current \\
\hline 8 & $\begin{array}{l}\text { Incline Creek (above Lakeshore Dr.) nr } \\
\text { Crystal Bay, Nev. }\end{array}$ & 10336700 & $\mathrm{~b}$ & $\begin{array}{l}\text { misc: } 67-69,74-75 \\
\text { gage: } 70-73,88 \text {-current }\end{array}$ & $\begin{array}{l}\text { field: } 70-73,78-81,88 \text {-current } \\
\text { wt: } 98 \text {-current }\end{array}$ & $\begin{array}{l}\text { n,p: } 70-73,78-80 \\
\text { n,p,i: } 88 \text {-current }\end{array}$ & $\begin{array}{l}\text { tot: } 70-73,78-80 \\
\text { sus: } 88 \text {-current }\end{array}$ \\
\hline 9 & $\begin{array}{l}\text { Incline Creek at Hwy. } 28 \text { at Incline } \\
\text { Village, Nev. }\end{array}$ & 103366995 & md & gage: 90 -current & field: 90-current & n,p,i: 90 -current & sus: 90 -current \\
\hline 10 & $\begin{array}{l}\text { Incline Creek abv Tyrol Village nr } \\
\text { Incline Village, Nev. }\end{array}$ & 103366993 & $\mathrm{ad}$ & gage: 90 -current & field: 90-current & n,p,i: 90 -current & sus: 90 -current \\
\hline 11 & $\begin{array}{l}\text { Incline Creek Tributary at County Club } \\
\text { Dr. nr Incline Village, Nev. }\end{array}$ & 103366997 & $\mathrm{~m}$ & misc: 89,91 -current & field: 89,91-current & $\begin{array}{l}\text { n,p,i: } 89 \\
\text { 91-current }\end{array}$ & sus: 89,91 -current \\
\hline 12 & Glenbrook Creek at Glenbrook, Nev. & 10336730 & $\mathrm{~b}$ & $\begin{array}{l}\text { misc: } 67-71 \\
\text { gage: } 72-75,88 \text {-current }\end{array}$ & $\begin{array}{l}\text { field: } 71-74,88 \text {-current } \\
\text { wt: } 98 \text {-current }\end{array}$ & $\begin{array}{l}\text { n,p: } 71-74,87 \\
\text { n,p,i: } 88 \text {-current }\end{array}$ & $\begin{array}{l}\text { tot: } 71-74,87 \\
\text { sus: } 88 \text {-current }\end{array}$ \\
\hline 13 & $\begin{array}{l}\text { Glenbrook Creek at Old Highway nr } \\
\text { Glenbrook, Nev. }\end{array}$ & 10336725 & $\mathrm{~m}$ & $\begin{array}{l}\text { misc: } 72-74,89 \\
91 \text {-current }\end{array}$ & $\begin{array}{l}\text { field: } 72-74,89, \\
\text { 91-current }\end{array}$ & $\begin{array}{l}\text { n,p: } 72-74,89 \\
91 \text {-current }\end{array}$ & $\begin{array}{l}\text { tot: } 72-74 \\
\text { sus: } 89,91 \text {-current }\end{array}$ \\
\hline 14 & $\begin{array}{l}\text { North Logan House Creek at Hwy. } 50 \\
\text { nr Glenbrook, Nev. }\end{array}$ & 10336735 & $\mathrm{~m}$ & misc: 91-current & field: 91-current & n,p,i: 91-current & sus: 91-current \\
\hline 15 & $\begin{array}{l}\text { Logan House Creek nr Glenbrook, } \\
\text { Nev. }\end{array}$ & 10336740 & $\mathrm{~b}$ & gage: 84 -current & field: 83-current & n,p,i: 88 -current & sus: $84-87,88$-current \\
\hline 16 & $\begin{array}{l}\text { Edgewood Creek at Lake Tahoe nr } \\
\text { Stateline, Nev. }\end{array}$ & 10336765 & m,ig & $\begin{array}{l}\text { gage: } 84-85,89-92 \\
\text { misc: } 93 \text {-current }\end{array}$ & field: $84-85,89$-current & $\begin{array}{l}\text { n,p: 84-85, } \\
\text { n,p,i: 89-current }\end{array}$ & sus: $84-85,89$-current \\
\hline 17 & $\begin{array}{l}\text { Edgewood Creek Tributary abv } \\
\text { Clubhouse nr Stateline, Nev. }\end{array}$ & $\begin{array}{l}385758- \\
119564401\end{array}$ & $\mathrm{u}$ & misc: 95 -current & field: 95-current & n,p,i: 95 -current & sus: 95-current \\
\hline 18 & $\begin{array}{l}\text { Culvert into Edgewood Creek abv } \\
\text { Hwy. } 50 \text { at Stateline, Nev. }\end{array}$ & $\begin{array}{l}385758- \\
119561101\end{array}$ & $\mathrm{u}$ & misc: 95 -current & field: 95-current & n,p,i: 95-current & sus: 95-current \\
\hline 19 & $\begin{array}{l}\text { Edgewood Creek (at Hwy. 50) at } \\
\text { Stateline, Nev. }\end{array}$ & 10336760 & $\mathrm{~b}$ & $\begin{array}{l}\text { misc: } 67-80 \\
\text { gage: } 93 \text {-current }\end{array}$ & field: $67-80,92$-current & n,p,i: 92 -current & sus: 92-current \\
\hline 20 & $\begin{array}{l}\text { Edgewood Creek at Palisade Dr. nr } \\
\text { Kingsbury, Nev. }\end{array}$ & 103367585 & md & gage: 90 -current & field: 90-current & $\mathrm{n}, \mathrm{p}, \mathrm{i}: 90$-current & sus: 90 -current \\
\hline 21 & Eagle Rock Creek nr Stateline, Nev. & 103367592 & $\mathrm{ad}$ & gage: 90 -current & field: 90-current & n,p,i: 90 -current & sus: 90 -current \\
\hline 22 & $\begin{array}{l}\text { Edgewood Creek Tributary nr Dagget } \\
\text { Pass, Nev. }\end{array}$ & 10336756 & m,ig & $\begin{array}{l}\text { gage: } 81-83 \\
\text { misc: } 91 \text {-current }\end{array}$ & field: 81-83,91-current & $\begin{array}{l}\text { n,p: } 81-83 \\
\text { n,p,i: } 91 \text {-current }\end{array}$ & sus: $81-83,91$-current \\
\hline 23 & $\begin{array}{l}\text { Edgewood Creek below South } \\
\text { Benjamin Dr. nr Dagget Pass, Nev. }\end{array}$ & 10336750 & $\mathrm{~m}$ & misc: 89,91 -current & field: 89,91-current & $\begin{array}{l}\text { n,p,i: } 89 \\
91 \text {-current }\end{array}$ & sus: 89,91 -current \\
\hline 24 & $\begin{array}{l}\text { Trout Creek nr mouth—east off } \\
\text { Bellevue/El Dorado Aves. }\end{array}$ & 10336795 & $\mathrm{t}$ & misc: 96-current & $\begin{array}{l}\text { field: } 96 \text {-current } \\
\text { wt: } 98 \text {-current }\end{array}$ & - & - \\
\hline 25 & $\begin{array}{l}\text { Trout Creek (at Hwy. 50) at South } \\
\text { Lake Tahoe, Calif. }\end{array}$ & 10336790 & $b, t$ & misc: 93-current & $\begin{array}{l}\text { field: } 72-74,89 \text {-current } \\
\text { wt: } 72-74,89-92,98 \text {-current }\end{array}$ & $\begin{array}{l}\text { n,p: } 79-85,87-92 \\
\text { n,p,i: } 93 \text {-current }\end{array}$ & $\begin{array}{l}\text { dai: } 72-74,81-85,88-92 \\
\text { sus: } 93 \text {-current }\end{array}$ \\
\hline 26 & $\begin{array}{l}\text { Trout Creek (at Martin Ave.) nr Tahoe } \\
\text { Valley, Calif. }\end{array}$ & 10336780 & $\mathrm{~m}, \mathrm{~g}, \mathrm{t}$ & gage: 61 -current & $\begin{array}{l}\text { field: } 61 \text {-current } \\
\text { wt: } 72-74,78,80-85,88 \text {, } \\
98 \text {-current } \\
\text { sc: } 81-83\end{array}$ & - & dai: $72-74,78,80-85,88$ \\
\hline 27 & $\begin{array}{l}\text { Cold Creek at mouth nr South Lake } \\
\text { Tahoe, Calif. }\end{array}$ & 10336779 & $\mathrm{t}$ & misc: 96-current & $\begin{array}{l}\text { field: } 96 \text {-current } \\
\text { wt: } 98 \text {-current }\end{array}$ & - & - \\
\hline
\end{tabular}


Table 1. Periods of record for streamflow, physical, nutrient, and sediment data collected at current U.S. Geological Survey/Lake Tahoe Interagency Monitoring Program stream network sites and other active USGS gaging stations in the Lake Tahoe Basin, California and Nevada-Continued

\begin{tabular}{|c|c|c|c|c|c|c|c|}
\hline $\begin{array}{l}\text { Site } \\
\text { no. }\end{array}$ & Site name & $\begin{array}{l}\text { Station } \\
\text { no. }\end{array}$ & $\begin{array}{l}\text { Site } \\
\text { type }\end{array}$ & Streamflow ${ }^{2}$ & Physical $^{3}$ & Nutrients ${ }^{4}$ & Sediment ${ }^{5}$ \\
\hline 28 & $\begin{array}{l}\text { Trout Creek at Pioneer Trail nr South } \\
\text { Lake Tahoe, Calif. }\end{array}$ & 10336775 & $\mathrm{md}, \mathrm{t}$ & gage: 90 -current & $\begin{array}{l}\text { field: } 90 \text {-current } \\
\text { wt: } 98 \text {-current }\end{array}$ & n,p,i: 90-current & sus: 90-current \\
\hline 29 & $\begin{array}{l}\text { Trout Creek at USFS Rd. 12N01 nr } \\
\text { Meyers, Calif. }\end{array}$ & 10336770 & $\mathrm{ad}, \mathrm{t}$ & gage: 90 -current & $\begin{array}{l}\text { field: } 90 \text {-current } \\
\text { wt: } 98 \text {-current }\end{array}$ & n,p,i: 90-current & sus: 90 -current \\
\hline 30 & $\begin{array}{l}\text { Upper Truckee River nr mouth off } \\
\text { Venice Dr. nr S Lake Tahoe, Calif. }\end{array}$ & 10336612 & $\mathrm{t}$ & - & $\begin{array}{l}\text { field: } 96 \text {-current } \\
\text { wt: } 98 \text {-current }\end{array}$ & - & - \\
\hline 31 & $\begin{array}{l}\text { Upper Truckee River (at Hwy. 50) at } \\
\text { South. Lake Tahoe, Calif. }\end{array}$ & 10336610 & $\mathrm{~b}, \mathrm{t}$ & $\begin{array}{l}\text { gage: } 72-74,77,78, \\
80 \text {-current }\end{array}$ & $\begin{array}{l}\text { field: } 72-74,78,80 \text {-current } \\
\text { wt: } 72-74,78,80-92,98 \text {-current } \\
\text { sc: } 81-83\end{array}$ & $\begin{array}{l}\text { n,p: } 77-92 \\
\text { n,p,i: } 93 \text {-current }\end{array}$ & $\begin{array}{l}\text { dai: } 72-76,78,81-92 \\
\text { sus: } 93 \text {-current }\end{array}$ \\
\hline 32 & $\begin{array}{l}\text { Upper Truckee River at Hwy. } 50 \text { below } \\
\text { Meyers, Calif. }\end{array}$ & 103366098 & $\mathrm{t}$ & misc: 96-current & $\begin{array}{l}\text { field: 96-current } \\
\text { wt: } 98 \text {-current }\end{array}$ & - & - \\
\hline 33 & $\begin{array}{l}\text { Upper Truckee River at Hwy. } 50 \text { above } \\
\text { Meyers, Calif. }\end{array}$ & 103366092 & md,t & $\begin{array}{l}\text { gage: } 90 \text {-current } \\
\text { misc: } 89\end{array}$ & $\begin{array}{l}\text { field: } 89,90 \text {-current } \\
\text { wt: } 98 \text {-current }\end{array}$ & $\begin{array}{l}\text { n,p,i: } 89 \\
90 \text {-current }\end{array}$ & sus: 89,90 -current \\
\hline 34 & Upper Truckee River nr Meyers, Calif. & 10336600 & m,ig & $\begin{array}{l}\text { gage: } 61-86 \\
\text { misc: } 96 \text {-current }\end{array}$ & field: $61-86,96$-current & - & - \\
\hline 35 & $\begin{array}{l}\text { Grass Lake Creek at Grass Lake Rd. nr } \\
\text { Meyers, Calif. }\end{array}$ & 10336593 & $\mathrm{t}, \mathrm{ig}$ & $\begin{array}{l}\text { gage: } 72-74 \\
\text { misc: } 96 \text {-current }\end{array}$ & $\begin{array}{l}\text { field: 72-74,96-current } \\
\text { wt: } 98 \text {-current }\end{array}$ & - & sus: $72-74$ \\
\hline 36 & $\begin{array}{l}\text { Upper Truckee River at South Upper } \\
\text { Truckee Rd nr Meyers, Calif. }\end{array}$ & 10336580 & $\mathrm{ad}, \mathrm{t}$ & gage: 90 -current & $\begin{array}{l}\text { field: } 90 \text {-current } \\
\text { wt: } 98 \text {-current }\end{array}$ & n,p,i: 90-current & sus: 90-current \\
\hline 37 & $\begin{array}{l}\text { General Creek (at Hwy. 89) nr Meeks } \\
\text { Bay, Calif. }\end{array}$ & 10336645 & $\mathrm{~b}$ & gage: 80 -current & $\begin{array}{l}\text { field: } 80 \text {-current } \\
\text { wt: } 81-92 \\
\text { sc: } 81-83\end{array}$ & $\begin{array}{l}\text { n,p: } 80-92 \\
\text { n,p,i: } 93 \text {-current }\end{array}$ & $\begin{array}{l}\text { dai: } 82-92 \\
\text { sus: } 93 \text {-current }\end{array}$ \\
\hline 38 & $\begin{array}{l}\text { Blackwood Creek (at Hwy. 89) nr } \\
\text { Tahoe City, Calif. }\end{array}$ & 10336660 & $\mathrm{~b}$ & gage: 61-current & $\begin{array}{l}\text { field: } 61 \text {-current } \\
\text { wt: } 75-78,80-92 \\
\text { sc: } 81-83\end{array}$ & $\begin{array}{l}\text { n,p: } 74-92 \\
\text { n,p,i: } 93 \text {-current }\end{array}$ & $\begin{array}{l}\text { dai: } 75-78,80-92 \\
\text { sus: } 93 \text {-current }\end{array}$ \\
\hline 39 & $\begin{array}{l}\text { Ward Creek at Hwy. } 89 \mathrm{nr} \text { Tahoe } \\
\text { Pines, Calif. }\end{array}$ & 10336676 & $\mathrm{~b}$ & gage: 73-current & $\begin{array}{l}\text { field: } 73 \text {-current } \\
\text { wt: } 73-78,79-92 \\
\text { sc: } 81-83\end{array}$ & $\begin{array}{l}\text { n,p: } 73-92 \\
\text { n,p,i: } 93 \text {-current }\end{array}$ & $\begin{array}{l}\text { dai: } 73-92 \\
\text { sus: } 93 \text {-current }\end{array}$ \\
\hline 40 & $\begin{array}{l}\text { Ward Creek at Stanford Rock Trail } \\
\text { crossing nr Tahoe City, Calif. }\end{array}$ & 10336675 & md & gage: 92-current & field: 91-current & n,p,i: 91-current & sus: 91-current \\
\hline 41 & $\begin{array}{l}\text { Ward Creek below confluence nr } \\
\text { Tahoe City, Calif. }\end{array}$ & 10336674 & $\mathrm{ad}$ & gage: 92-current & field: 91-current & n,p,i: 91-current & inst: 91-current \\
\hline \multicolumn{8}{|c|}{ Other active U.S. Geological Survey sites } \\
\hline 42 & Marlette Lake near Carson City, Nev. & 10336710 & $\mathrm{~g}$ & lake level: 74-current & - & - & - \\
\hline 43 & Marlette Creek near Carson City, Nev. & 10336715 & $\mathrm{~g}$ & gage: 74-current & field: 74-current & - & - \\
\hline 44 & Lake Tahoe at Tahoe City, Calif. & 10337000 & g & lake level: 1900-current & chem: $69,78,79$ & - & - \\
\hline 45 & Truckee River at Tahoe City, Calif. & 10337500 & g & $\begin{array}{l}\text { gage: } 1895-1896, \\
\text { 1900-current }\end{array}$ & $\begin{array}{l}\text { field: } 1900 \text {-current } \\
\text { wt: } 93-94\end{array}$ & - & - \\
\hline
\end{tabular}

\footnotetext{
1 Site type: b, base (near mouth); md, mid-development; ad, above-development; m, miscellaneous; g, other active gage; ig, inactive gage; $\mathrm{t}$, water temperature; $\mathrm{u}$, urban runoff.

2 Streamflow: misc, instantaneous flow measurements only; gage, flow measurements, continuous recording gage, and daily flows.

3 Physical: field, field measurements; wt, daily water-temperature record; sc, daily specific-conductance record; chem, chemical.

4 Nutrients: n, nitrogen; p, phosphorus; i, iron.

5 Sediment: tot, total; sus, suspended; dai, daily; inst, instantaneous.
}

sheds in 1988 and expanded to 13 sites in 7 watersheds in 1990. The number of stations in the network was increased again in 1991, 1993, and 1996, adding 21 additional sampling sites in 7 additional watersheds. The purpose of expanding the data network was to support the assessments of the effects of land use and development in the Lake Tahoe Basin on water quality.

In 1998, a 12-site water-temperature (WT) monitoring network (7 existing and 5 new sites) was installed in the Upper Truckee River and Trout Creek watersheds. In 1998, WT monitors were added to Incline Creek near Crystal Bay, Nev., and Glenbrook Creek at Glenbrook, Nev. The purpose of the WT network is to provide information for groundwater/surface-water interaction studies and for manmade organics studies.
The current LTIMP sampling network includes 32 tributary sites in 14 Lake Tahoe watersheds in California and Nevada (fig. 1) and was described previously by Boughton and others (1997) and Rowe and Stone (1997). Table 1 describes the period of record for current and historical data collection for the 34 sites plus 2 other active USGS streamflow gage sites, 2 lake level gage sites, 5 USGS water temperature only sites, and 2 urban-runoff sites. The longest periods of record are 1900current for lake levels of Lake Tahoe, 1961-current for streamflow, 1961-current for water-quality field measurements, and 1973-current for nutrients and sediment.

Current collection in the LTIMP program is streamflow, physical, sediment, and nutrient data (table 2) and is divided into three sampling schedules. The primary sites (table 1) include continuous streamflow gages and about 30-40 samples collected per year at 10 base (near- 


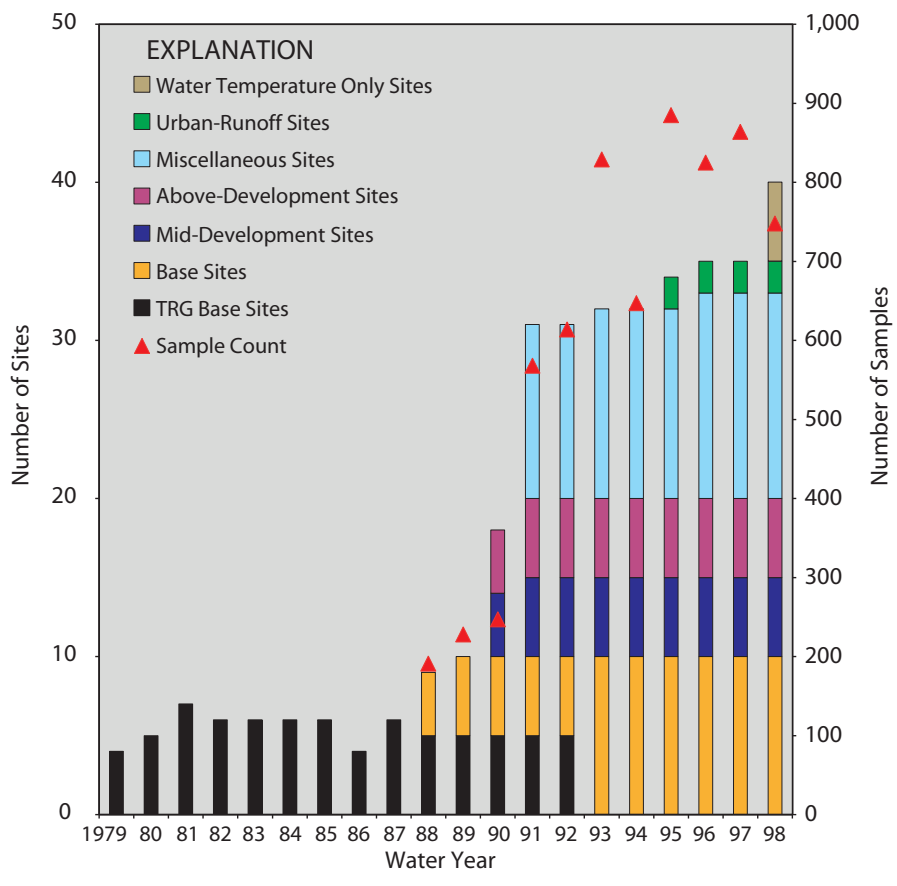

Figure 2. History of Lake Tahoe Interagency Monitoring Program in Lake Tahoe Basin, California and Nevada. (See Table 1 footnotes for site definition.)

mouth) sites in 10 watersheds in California and Nevada. The secondary sites (table 1) include continuous streamflow gages and about 25 samples collected per year at the 10 mid- and above-development sites on 5 of the 14 watersheds in California and Nevada. Throughout the year, collection of routine monthly baseline sampling with an extra emphasis on snow-melt runoff and storm runoff periods is done at the primary and secondary sites. The remaining 14 miscellaneous sites (table 1) are monitored but ungaged in Nevada. Data collection on these sites includes 6-10 samples per year, mainly during snow-melt runoff and storm-runoff events.

\section{-Timothy G. Rowe}

\section{REFERENCES CITED}

Boughton, C.J., Rowe, T.G., Allander, K.K., and Robledo, A.R., 1997, Stream and ground-water monitoring program, Lake Tahoe Basin, Nevada and California: U.S. Geological Survey Fact Sheet FS-100-97, 6 p.

Byron, E.R., and Goldman, C.R., 1989, Land-use and waterquality in tributary stream of Lake Tahoe, California-Nevada: Journal of Environmental Quality, v. 18, no. 1, p. 84-88.

Goldman, C.R., and Byron, E.R., 1986, Changing water quality at Lake Tahoe-The first five years of the Lake Tahoe Interagency Monitoring Program: Tahoe Research Group, Institute of Ecology, University of California, Davis, 12 p.

Rowe, T.G. and Stone, J.C., 1997, Selected hydrologic features of the Lake Tahoe Basin, California and Nevada: U.S. Geological Survey Open-File Report 97-384, 1 sheet.
Table 2. Lake Tahoe Interagency Monitoring Program stream network sample analysis

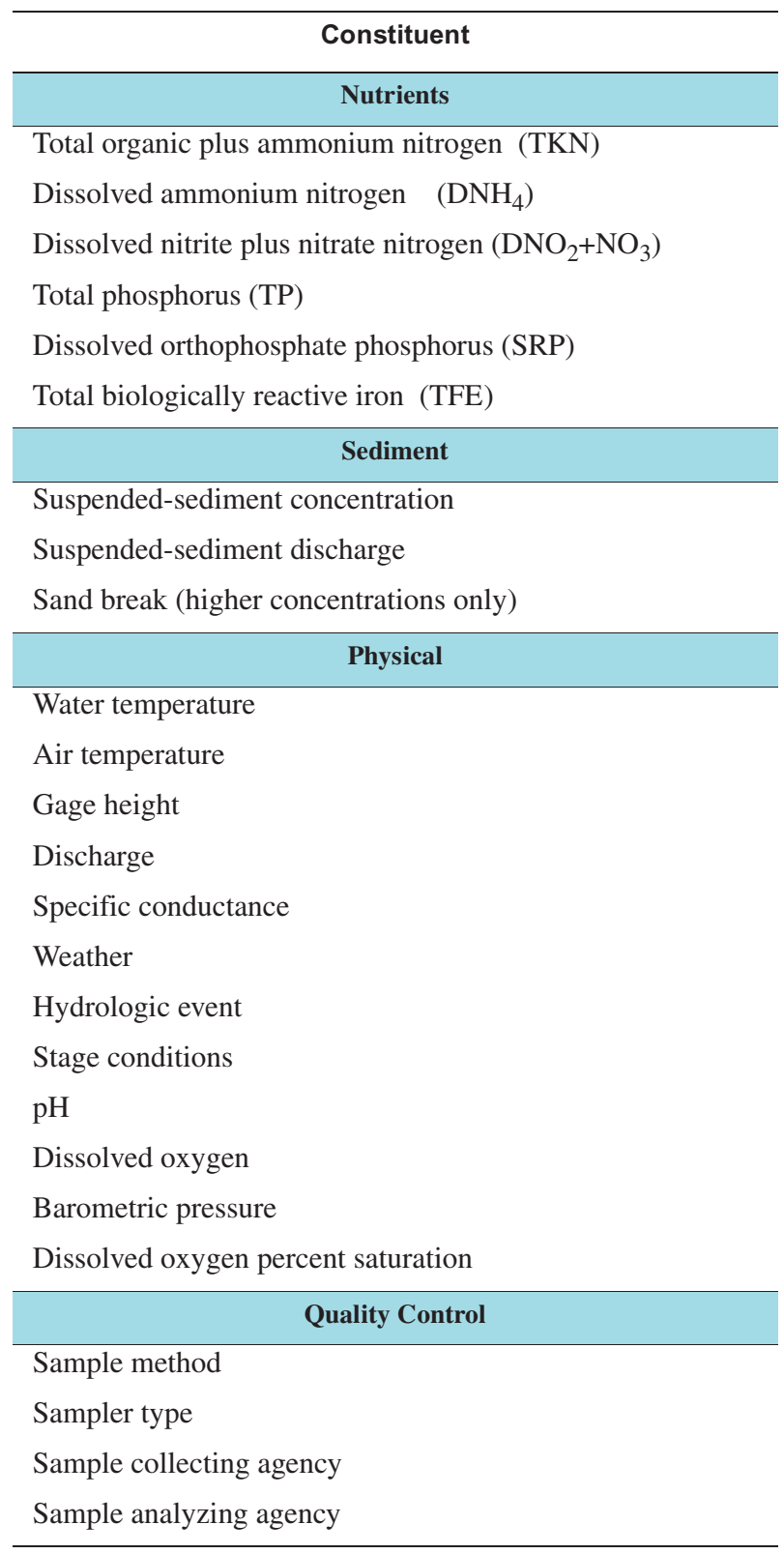

\section{For further information contact:}

Public Information Assistant

U.S. Geological Survey

333 W. Nye Lane

Carson City, NV 89706

(775) 887-7649

Email: GS-W-NVpublic-info@usgs.gov

Nevada District homepage: http://nevada.usgs.gov

Lake Tahoe Clearinghouse homepage: http://tahoe.usgs.gov 